\section{On Determining the Packed Cell Volume}

\author{
H. E. HUTCHISON \\ From the Department of Haematology, University \\ and Western Infirmary, Glasgow
}

(RECEIVED FOR PUBLICATION APRIL 30, 1960)

The technical simplicity of estimating the packed cell volume seems to arouse a false sense of security in its reproducibility. It is, for example, generally held by clinicians to be less subject to technical artefact than measurement of $\mathrm{Hb}$ concentration. Yet it has been experience here that, when the M.C.H.C. of the same patient has been determined two or three times a week over a month or so and fluctuations have occurred, they have been due to a variation in the figure recorded for the P.C.V. and not in the $\mathrm{Hb}$ concentration. Since becoming conscious of the possibility of significant errors in P.C.V. determination as ordinarily carried out, two main sources of error have been found, namely, errors in reading the haematocrit tube, and errors through failing to achieve uniform and adequate packing of the red cells.

In the actual reading of the haematocrit tube, two important points emerged after watching several technicians reading the same tube. First, there were variations in the angle (to the vertical) at which the tube was held; these were overcome by using the Wintrobe stand to keep the haematocrit tube vertical. Secondly, the angle to the horizontal at which the reader observed the top of the packed cell layer varied. The ideal position is to have the reader's eye at the same level as the top of the packed cell layer. Magnification, by enabling the reader to line up the back with the front of the buffy coat when taking the reading, ensures a close approximation to the ideal level. In addition, magnification allows evaluation of the level of the packed cell layer to within half or quarter of a division; it also becomes possible to make an approximate allowance in admixture of red with the white cells, when, as sometimes happens, separation of the two is not sharp. Magnification therefore adds precision to the reading.

Failure to achieve uniform and adequate packing of the red cells is the second important source of error, and may result from use of several different centrifuges for P.C.V. determination in the same laboratory, for they are not equally effective in producing the necessary centrifugal force. Even a centrifuge designed expressly for the determination of the P.C.V. may be fundamentally unsuitable for the purpose and, as an illustration of this, experience in this laboratory with the M.S.E. haematocrit centrifuge is revealing.

According to Wintrobe (1956), 30 minutes at a force of $2,264 \mathrm{G}$ is required to produce so-called "complete" packing, i.e., more time at this force will not produce further packing. The M.S.E. haematocrit centrifuge at its geverned speed of 3,000 r.p.m. exerts only an R.C.F. of $1,360 \mathrm{G}$, for the radius of the centrifuge is only $13.5 \mathrm{~cm}$., and to produce $2,264 \mathrm{G}$ a speed of 3,800 r.p.m. would be required. The machine cannot reach this speed even following maximum adjustment by the works engineer. The maximum speed is 3,375 revs. and at this it develops an R.C.F. of only $1,780 \mathrm{G}$, which is still far short of the 2,264 G specified by Wintrobe. In brief, the centrifuge has been wrongly designed. The radius is too short, being only $13.5 \mathrm{~cm}$. instead of $22.5 \mathrm{~cm}$.

If these conclusions regarding the M.S.E. haematocrit centrifuge are correct, packing should be incomplete at $30 \mathrm{~min}$., the period recommended by the manufacturers, and this proves to be so. Thus, if after reading the P.C.V. the tubes are respun for a further $30 \mathrm{~min}$. a significant increase in packing is obtained, especially obvious, of course, with blood samples in which the P.C.V. is not greatly reduced. Secondly, since packing is incomplete, the M.C.H.C. level should tend to be generally a little lower than the normal $32-36 \%$, particularly when normal range $\mathrm{Hb}$ concentrations are considered, as these will have a P.C.V. in the $40 \%$ range. What is found is as shown in the table.

M.C.H.C. IN 50 BLOOD SAMPLES WITH Hb CONCENTRATION OF $96 \%$ HALDANE OR OVER

\begin{tabular}{r|c}
\hline M.C.H.C. & \% of Cases \\
\hline Less than 31 & 12 \\
31 & 32 \\
32 & 38 \\
33 & 14 \\
34 & 2 \\
More than 34 & 2 \\
\hline
\end{tabular}

\section{Comment}

Less care than is required is usually given to reading the P.C.V. tubes. Indeed, the subject is not mentioned in any detail in most standard texts as being worthy of special consideration, and experience here illustrates the fallacy in this common attitude. Nor is it customary to consider the force applied by the centrifuge in G. Instead, the figure of 3,000 r.p.m. for $30 \mathrm{~min}$. is too often all that is thought important. That this should occur even in the case of a centrifuge designed expressly for determining the P.C.V. is an indication of how widespread this erroneous impression may be.

I am indebted to Dr. A. Thom, Department of Engineering, University of Glasgow, for his surveillance of the R.C.F. calculations, etc., quoted.

\section{REFERENCE}

Wintrobe, M. M. (1956). In Clinical Haematology, 4th ed., pp. 367369. Kimpton, London. 